\title{
Original
}

\section{N-methyl-D-aspartate Receptor Mediates X-irradiation-induced Drebrin Decrease in Hippocampus}

\author{
Shuchuan Miao ${ }^{1}$, Noriko Koganezawa ${ }^{1}$, Kenji Hanamura ${ }^{1}$, Anggraeini Puspitasari ${ }^{1,2}$ and \\ Tomoaki Shirao ${ }^{1}$ \\ 1 Department of Neurobiology and Behavior, Gunma University Graduate School of Medicine, 3-39-22 Showa-machi, Maebashi, Gunma 371- \\ 8511, Japan \\ 2 International Open Laboratory, Harvard Medical School/MGH Dr. Held Lab, Gunma University Initiative for Advanced Research (GIAR), \\ 3-39-22 Showa-machi, Maebashi, Gunma 371-8511, Japan
}

\begin{abstract}
Background \& Aims: Therapeutic X-irradiation of brain possibly causes cognitive impairment associated with synaptic dysfunction. Drebrin is a postsynaptic actin-binding protein and plays an important role for learning and memory. We have recently reported that drebrin decreases transiently in the molecular layer of dentate gyrus (MLDG) of hippocampus after X-irradiation in parallel with fear memory impairment. However, the mechanism regulating the drebrin decrease is not clarified. Our previous study has shown that activation of N-methyl-D-aspartate (NMDA) receptors induces transient drebrin exodus from dendritic spines. In the present study, we examined whether NMDA receptor relates to X-irradiation-induced drebrin decrease. Methods: Ten-week-old male mice, pretreated with an NMDA receptor antagonist MK801, were exposed to $10 \mathrm{~Gy}$ of whole brain X-irradiation and fixed after 8 hours. Immunostaining intensity of drebrin and PSD-95 in MLDG and number of doublecortin (DCX) positive neurons in dentate gyrus (DG) were analyzed. Results: X-irradiation decreased the intensity of drebrin and doublecortin-positive neurons. MK801 inhibited the decrease of drebrin intensity, but not the decrease of DCX-positive neurons. PSD-95 intensity did not change after irradiation. Conclusion: Our results indicate that NMDA receptor mediates the X-irradiation-induced decrease of drebrin, and suggest that NMDAR-mediated drebrin decrease underlies X-irradiation-induced acute transient cognitive impairment.
\end{abstract}

\begin{tabular}{l} 
Article Information \\
\hline Key words: \\
Drebrin, \\
NMDA receptor, \\
PSD-95, \\
Doublecortin, \\
X-irradiation \\
\hline Publication history: \\
Received: December 11, 2017 \\
Accepted: December 15, 2017 \\
\hline Corresponding author: \\
Tomoaki Shirao \\
Department of Neurobiology and Behavior, Gunma \\
University Graduate School of Medicine, 3-39-22 Showa- \\
machi, Maebashi, Gunma 371-8511, Japan \\
Tel: +81-27-220-8050 \\
E-mail: tshirao@gunma-u.ac.jp \\
\hline
\end{tabular}

\section{Introduction}

Brain tumors are common tumors in childhood and adolescence. ${ }^{1}$ Radiation therapy is an important treatment of the brain tumor. Although the survival rate has been increased, radiation induced cognitive impairment ${ }^{2,3}$ is a serious side effect. At present, the underlying mechanism of the cognitive impairment is poorly understood.

Although most studies in recent years focused on late delayed effects of irradiation, we have recently reported that 10 Gy of whole brain X-irradiation causes acute (within 24 hours) and transient fear memory impairment. In parallel with fear memory impairment, drebrin, a postsynaptic actin binding protein, transiently decreases in molecular layer of dentate gyrus (MLDG) of hippocampus after X-irradiation. ${ }^{4}$ Drebrin plays an important role for the synaptic plasticity ${ }^{5-7}$ and its expression is decreased in Alzheimer's disease and Down syndrome. ${ }^{8-10}$ These indicate that drebrin decrease is closely related to the underlying mechanism of cognitive impairment after X-irradiation. However, it is still unknown what mediates the acute decrease of drebrin after X-irradiation.

We have previously shown that glutamate stimulation causes the exodus of drebrin from dendritic spines to the shaft in vitro and N-methyl-D-aspartate (NMDA) receptor antagonist AP5 inhibits the drebrin exodus. ${ }^{11,12}$ This raised the possibility that NMDA receptors are 


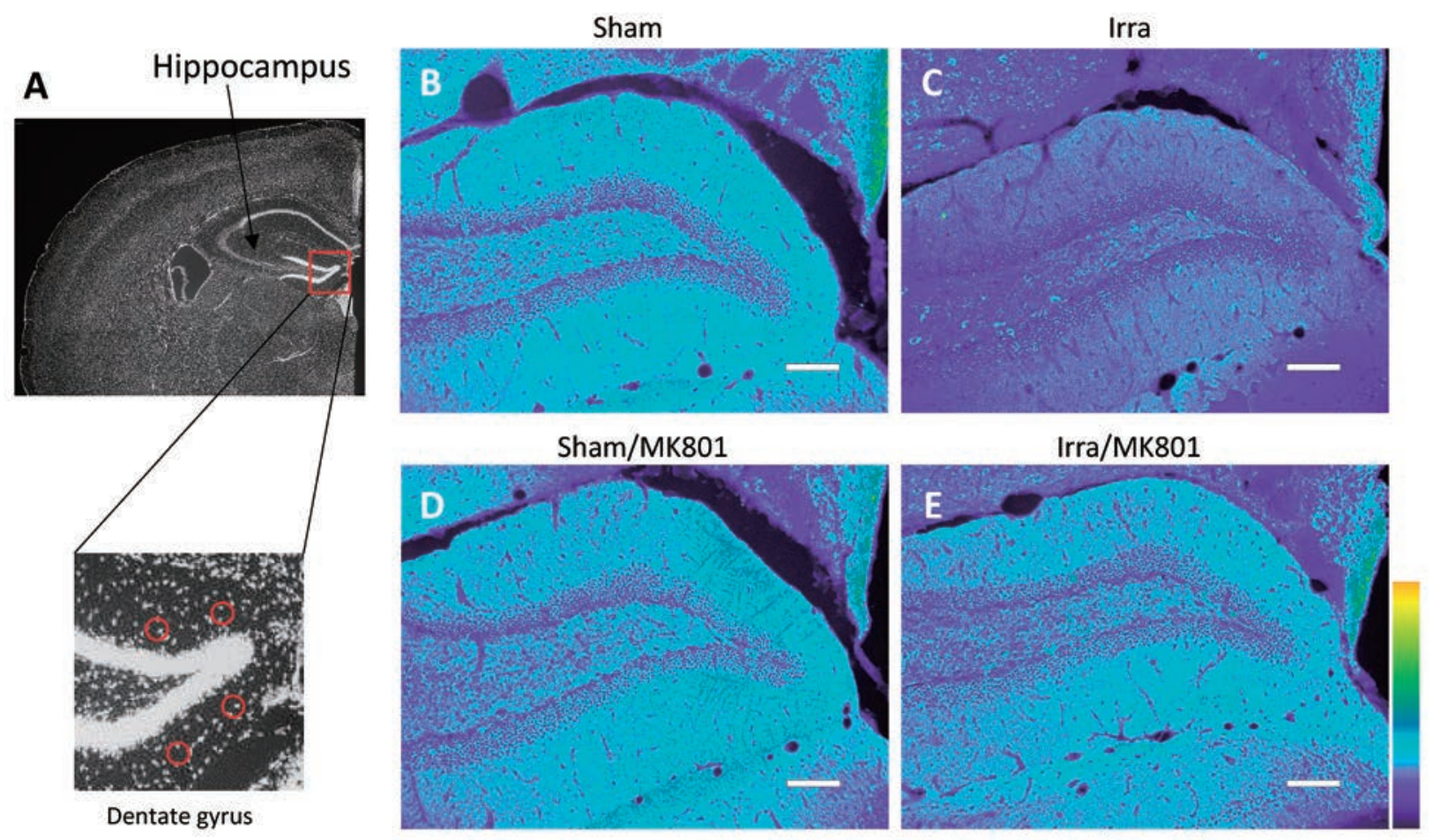

Fig. 1 MK801 inhibited X-irradiation-induced decrease of drebrin intensity in molecular layer of dentate gyrus (DG) of hippocampus.

(A) Typical hippocampal slice image labeled with 4', 6-diamidino-2-phenylindole, comparable to that of the mouse brain atlas, ${ }^{13}$ at the bregma $-1.7 \mathrm{~mm}$, coronal section. The red rectangle represents the position of DG shown in the left bottom at higher magnification. Four random regions of interest were selected (red circles, diameter $33 \mu \mathrm{m}$ ) in the molecular layer of DG to measure the intensity. (B-E) Representative pseudo color images from fluorescent images of drebrin immunostaining in DG from brain sections of Sham, irradiated (Irra), Sham/MK801 and Irra/MK801 mice. Note that the immunostaining intensity in C is weaker than those in B, D and E. Color bar: the intensity increases from purple to yellow. Scale bars $=100 \mu \mathrm{m}$.

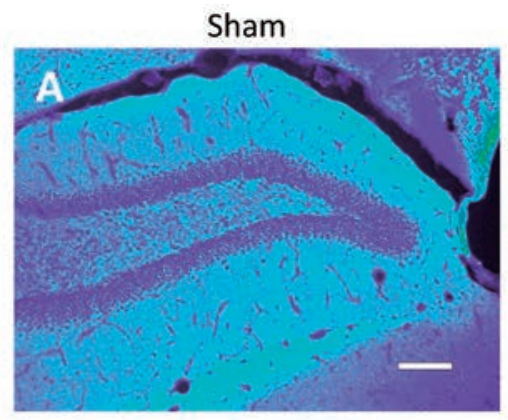

Sham/MK801

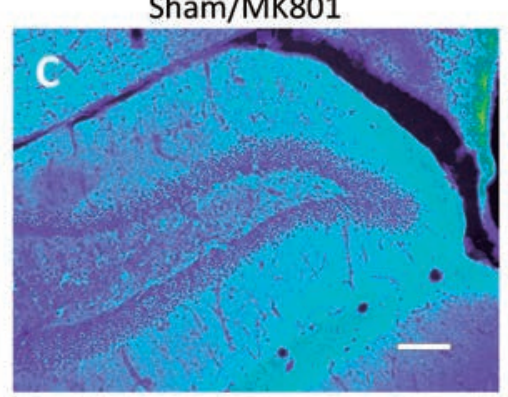

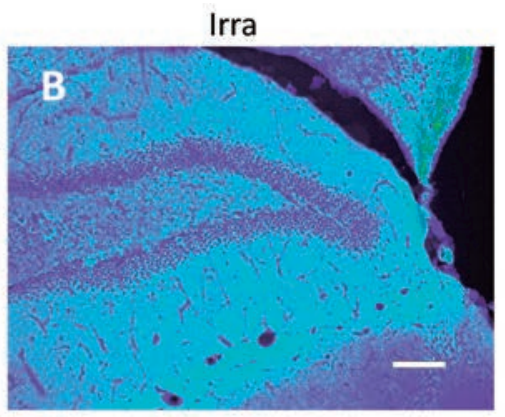

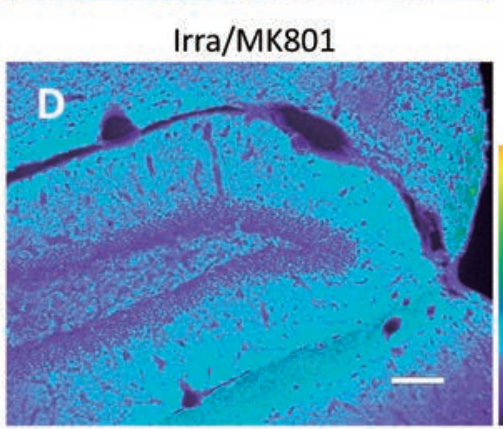

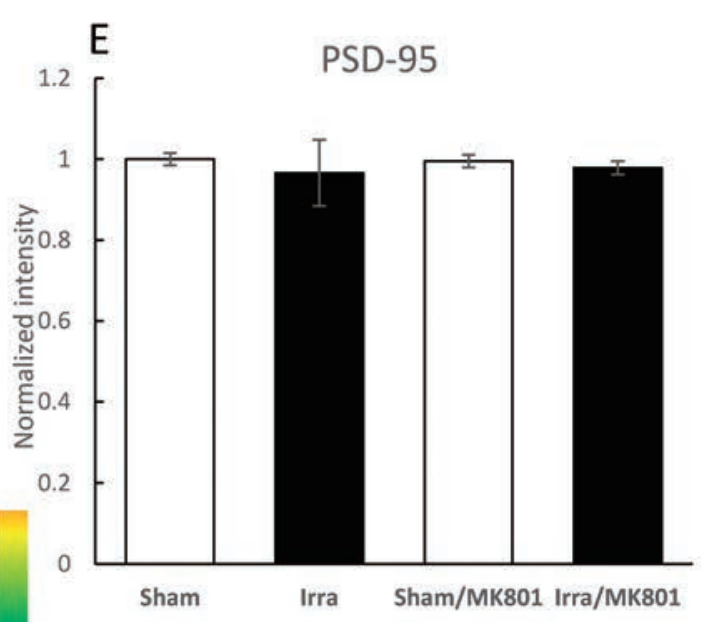


involved in the X-irradiation-induced drebrin decrease in MLDG of the hippocampus. In this study, we irradiated mouse whole brains and compared the decrease of drebrin immunostaining intensity in MLDG between mice with and without the pretreatment of an NMDA receptor antagonist MK801.

\section{Materials and Methods}

\section{1-1. Animals and $X$-irradiation}

All animal experiments were performed following guidelines of the Animal Care and Experimentation Committee of Gunma University. Efforts were made to minimize the number of animals used and their pain and discomfort. Ten-week-old male mice (C57BL/6N, Japan SLC Inc., Shizuoka, Japan) were injected with MK801 (2 mg/kg, i.p, Sigma-Aldrich, St. Louis, MO) or the same volume of saline 10 minutes before whole brain $\mathrm{X}$-irradiation. Mice were anesthetized using 2.5\% tribromoethanol $(250 \mathrm{mg} / \mathrm{kg}$, Sigma-Aldrich) and irradiated with 10 Gy X-irradiation $(1.3 \mathrm{~Gy} / \mathrm{min}$, Shimadzu X-TITAN 225S X-ray generator, Shimadzu Inc., Kyoto, Japan).

\section{1-2. Immunohistochemistry}

Eight hours after irradiation, mice were anesthetized and perfused with $4 \%$ paraformaldehyde in $0.1 \mathrm{M}$ phosphate buffer ( $\mathrm{pH}$ 7.4). Brain tissue was removed and transferred into $30 \%$ sucrose in PBS. Frozen tissue was cut into $20 \mu \mathrm{m}$ thick coronal sections using Leica CM3050 S Research Cryostat (Leica Microsystems, Wetzlar, Germany). A group of four slices from each condition was put on a glass slide for comparison. Slices were permeabilized with $0.1 \%$ Triton X-100 and incubated with $3 \%$ bovine serum albumin in PBS. They were immunostained with a rabbit polyclonal antibody against drebrin A (DAS2, 1:200; Immuno-Biological Laboratories Co., Maebashi, Japan), a rabbit polyclonal antibody against PSD-95 (1:500; Zymed Laboratories, San Francisco, CA) and a goat polyclonal antibody against doublecortin (DCX, 1:200; Santa Cruz Biotechnology, Dallas, TX) separately in $4{ }^{\circ} \mathrm{C}$ for overnight. After washing with PBS, they were immunostained with Alexa 488-conjugated anti-rabbit IgG antibody (Thermo Fisher Scientific Inc., Waltham, MA) and FITC-conjugated anti-goat IgG antibody (Santa Cruz Biotechnology). Nuclei were labeled using 4', 6-diamidino-2-phenylindole. They were mounted with PermaFluor (Lab Vision Co., Fremont, CA) before image capture.

\section{1-3. Image capture and analysis}

The images were obtained with an Axio Imager Z2 microscope (Zeiss, Jena, Germany). Fluorescence images were analyzed using MetaMorph software (Molecular Devices, Sunnyvale, CA).

\section{1-4. Quantification and statistical analysis}

Four regions of interest in MLDG of hippocampus were chosen randomly. The immunostaining intensity of the regions were automatically obtained. DCX posi- tive neurons in dentate gyrus (DG) were counted manually. Data were presented as the mean \pm SEM and were analyzed by two-way ANOVA followed by Scheffe's F test using SPSS software (IBM Corp., version 22.0., New York, NY).

\section{Results}

1-1. NMDA receptor antagonist MK801 inhibited X-irradiation-induced decrease of drebrin intensity

We irradiated the whole brain of mice with 10 Gy $\mathrm{X}$-irradiation and analyzed the intensity of drebrin in MLDG (Fig. 1). Regions of interest were chosen as in Fig. 1A. The drebrin intensity of each group was normalized using the average intensity of sham mice without MK801 as previously. ${ }^{4}$ As shown in Fig. 1B, C and Fig. 2, the intensity of drebrin after $10 \mathrm{~Gy}$ X-irradiation without MK801 decreased significantly compared to the sham group $(\mathrm{p}<0.01)$, which was consistent with our previous result. ${ }^{4}$

To examine whether NMDA receptors are involved in the X-irradiation-induced drebrin decrease, we pretreated mice with MK801, an NMDA receptor antagonist, 10 minutes before X-irradiation. As shown in Fig. 1D, E and Fig. 2, there was no difference of drebrin intensity between sham and X-irradiated groups when MK801 was prsent. This suggests that MK801 has a protective effect on X-irradiation-induced decrease of drebrin immunostaining intensity.

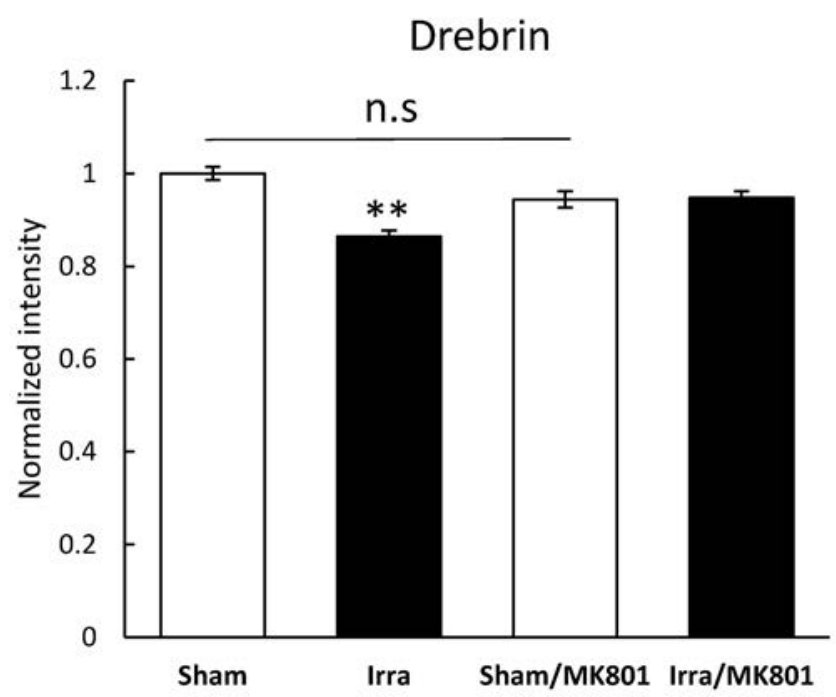

Fig. 2 Quantification of normalized drebrin intensity. Sham, $\mathrm{n}=32$ slices from 6 brains; Irra, $\mathrm{n}=28$ slices from 6 brains; Sham/MK801, $n=31$ slices from 6 brains; Irra/ MK801, $\mathrm{n}=29$ slices from 6 brains. Data are presented as the mean \pm SEM, and are analyzed with ANOVA, followed by Scheffe's F test. **p $<0.01$, vs Sham, Sham/ MK801 and Irra/MK801. n.s., no significance. 

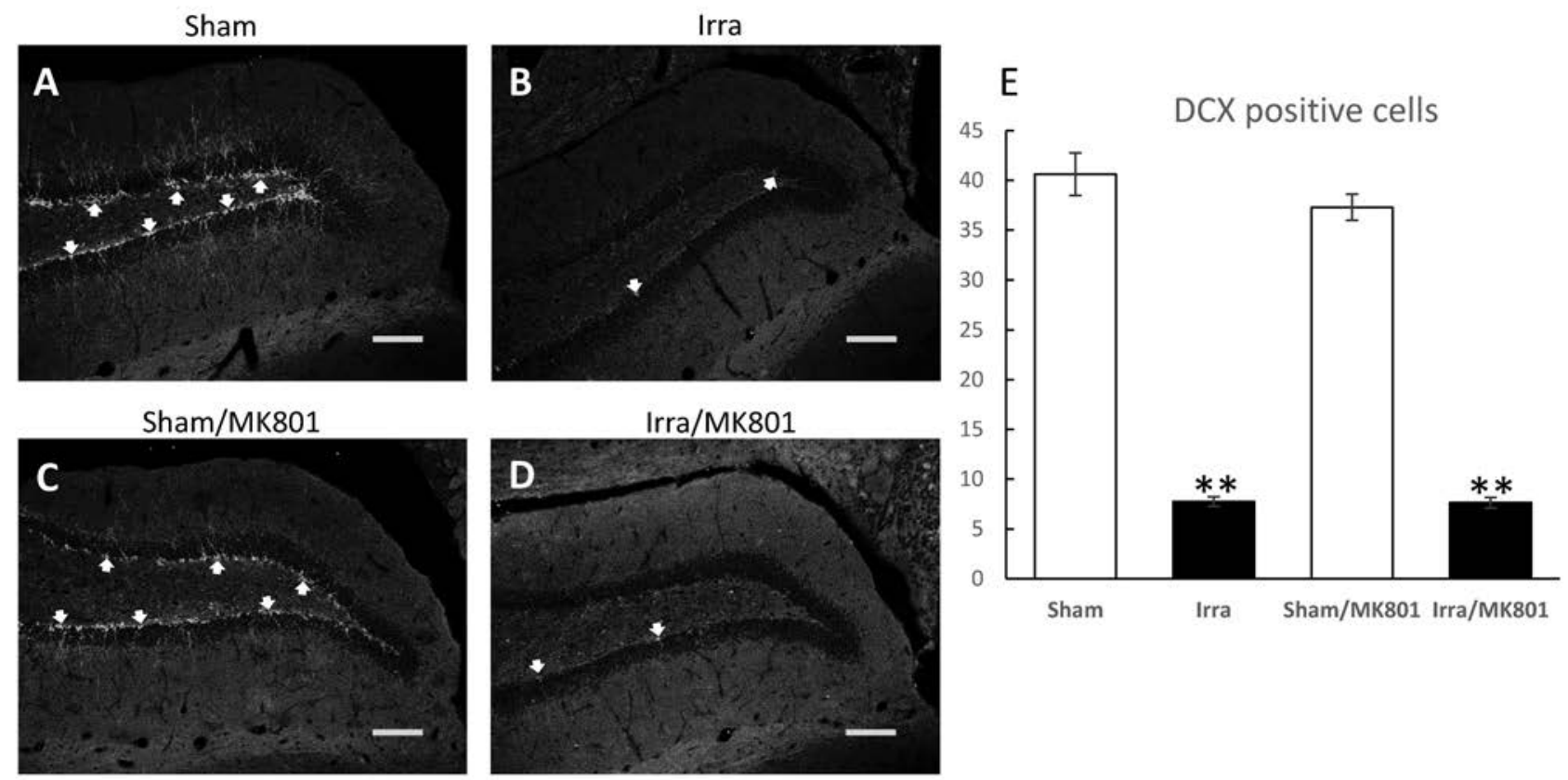

Fig. 4 MK801 did not affect the X-irradiation-induced decreases of DCX positive neurons in dentate gyrus (DG) of hippocampus.

(A-D) Representative fluorescent images of doublecortin (DCX) immunostaining in DG from the sections of Sham, irradiated (Irra), Sham/MK801 and Irra/MK801 mice. Arrows indicate DCX positive neurons. (E) Quantification of DCX positive neurons. Sham, $n=26$ slices from 3 brains; Irra, $n=26$ slices from 3 brains; Sham/MK801, $n=24$ slices from 3 brains; Irra/MK801, $n=24$ slices from 3 brains. Data are presented as the mean \pm SEM, and are analyzed with ANOVA, followed by Scheffe's F test. **p $<$ 0.01, Sham vs Irra, Sham /MK801 vs Irra /MK801. Scale bars $=100 \mu \mathrm{m}$.

\section{1-2. Postsynaptic protein PSD-95 was not affected by X-irradiation}

Because drebrin is specifically concentrated in dendritic spines, we examined whether PSD-95, another postsynaptic protein, was also decreased after X-irradiation or not. PSD-95 is a member of a membrane-associated guanylate kinase family, and, like drebrin, plays an important role in synaptic plasticity and stabilization of synaptic changes during long-term potentiation. ${ }^{14,15}$ Different from drebrin immunostaining intensity, PSD-95 immunostaining intensity did not change after X-irradiation (Fig. 3A, B and E). Pretreatment with MK801 did not affect the intensity of PSD-95 either in sham group or X-irradiated group (Fig. 3C-E). This indicates that the drebrin decrease at 8 hours after $10 \mathrm{~Gy} \mathrm{X}$-irradiation is not because of the disruption of dendritic spine structure but of the specific disappearance of drebrin from dendritic spines.

\section{1-3. MK801 did not affect $X$-irradiation-induced decrease of DCX positive neurons in the DG of hippocampus}

Finally, we confirmed whether MK801 affected $\mathrm{X}$-irradiation-induced drebrin decrease specifically. We have previously shown that number of DCX-positive cells (newly generated neurons) in DG decreased at 8 hours after 10 Gy X-irradiation. ${ }^{4}$ Thus we immunostained mice brains and quantified the number of DCX positive neurons to investigate if MK801 also has an effect on the cell death caused by X-irradiation. X-irradiation significantly decreased DCX positive neurons (arrows in Fig. 4A-D), and MK801 pretreatment did not inhibit the decrease (Fig. 4C-E). This indicates that MK801 does not have protective effects on cell death of newly generated neurons.

\section{Discussion}

The present study indicates that X-irradiation activates NMDA receptor resulting in the drebrin decrease. However, NMDA receptor activation induced by X-irradiation does not relate to either PSD-95 immunostaining intensity or the cell death of newly generated neurons. Therefore, NMDA receptor seems to specifically affect a drebrin-specific phenomenon, resulting in the drebrin decrease in MLDG.

Drebrin exodus is a drebrin-specific phenomenon induced by NMDA receptor activation. During drebrin exodus, ${ }^{12}$ drebrin distribution pattern transiently changes from dendritic spine localization into the diffuse distribution within dendrites and cell soma, without changing drebrin expression level. ${ }^{11}$ This causes the decrease of dot-like drebrin immunostaining pattern in neuropil region. This indicates that $\mathrm{X}$-irradiation transiently decreases drebrin immunostaining intensity in MLDG through drebrin exodus induced by NMDA receptor activation.

Another possible mechanism is the decrease of drebrin expression. However, we have previously shown that X-irradiation does not change drebrin expression level ${ }^{4}$ although the drebrin immunostaining in MLDG of the hippocampus is decreased. 
We have previously shown that the intensity of presynaptic marker synapsin I in MLDG does not change within 24 hours after $10 \mathrm{~Gy} \mathrm{X}$-irradiation. ${ }^{4}$ Synapsin I is present in the nerve terminal of axons and implicated in the modulation of neurotransmitter release. Thus the release of transmitters from presynaptic structures may not be altered in an early phase after X-irradiation.

In vitro experiments indicated that glutamate uptake is increased in NTera 2/cl.D1-derived neurons ${ }^{16}$ while it is decreased in NTera 2/cl.D1-derived astrocytes, after gamma irradiation or high charge energy particle irradiation. $^{17,18}$ Therefore the transport of glutamate may take part in synaptic pathology after irradiation by changing the extracellular glutamate level. It would be interesting to examine whether the irradiation-induced change of the glutamate transporter is related to the decrease of drebrin immunostaining intensity in vivo and in vitro.

Besides, whole brain X-irradiation induces neuroinflammation and blood-brain barrier disruption in brain., 3,19 It is reported that the expression of several inflammation factors, such as tumor necrosis factor- $\alpha$, interleukin- $1 \beta$, interleukin- 6 and cyclooxygenase- 2 are increased after $\mathrm{X}$-irradiation in vivo and in vitro. ${ }^{20,21}$ These inflammation factors might cause NMDA receptor activation, resulting in drebrin exodus.

Shi et al. have reported that the expression of NR1 and NR2A, but not NR2B, in CA1 of rat hippocampus is increased 12 months after fractionated whole-brain X-irradiation. $^{22}$ Thus, the expression of NMDA receptor subunit could be changed also in the acute phase after $\mathrm{X}$-irradiation, resulting in the NMDA receptor activation.

In conclusion, the present study indicates that NMDA receptor mediates $X$-irradiation-induced drebrin decrease possibly via drebrin exodus, and suggests that NMDAR-mediated drebrin decrease underlies X-irradiation-induced acute transient cognitive impairment. Blocking NMDA receptors might be potential therapy for X-irradiation-induced cognitive impairment.

\section{Acknowledgements}

This research was supported by the Gunma University Program for Cultivating Global Leaders in Heavy Ion Therapeutics and Engineering, by KAKENHI Grant Numbers 19200029, 15K14344, 16K18376 and 17H03557 from JSPS and by AMED in Japan.

\section{Reference}

1. Lee YW, Cho HJ, Lee WH, et al. Whole brain radiation-induced cognitive impairment: pathophysiological mechanisms and therapeutic targets. Biomol Ther (Seoul) 2012; 20: 357370 .

2. Greene-Schloesser D, Moore E, Robbins ME. Molecular pathways: radiation-induced cognitive impairment. Clin Cancer Res 2013; 19: 2294-2300.
3. Makale MT, McDonald CR, Hattangadi-Gluth JA, et al. Mechanisms of radiotherapy-associated cognitive disability in patients with brain tumors. Nat Rev Neurol 2017; 13: 52-64.

4. Puspitasari A, Koganezawa N, Ishizuka Y, et al. X irradiation induces acute cognitive decline via transient synaptic dysfunction. Radiat Res 2016; 185: 423-430.

5. Kojima N, Yasuda H, Hanamura K, et al. Drebrin A regulates hippocampal LTP and hippocampus-dependent fear learning in adult mice. Neuroscience 2016; 324: 218-226.

6. Koganezawa N, Hanamura K, Sekino Y, et al. The role of drebrin in dendritic spines. Mol Cell Neurosci 2017; 84: 85-92.

7. Sekino Y, Koganezawa N, Mizui T, et al. Role of drebrin in synaptic plasticity. Adv Exp Med Biol 2017; 1006:183-201.

8. Harigaya Y, Shoji M, Shirao T, et al. Disappearance of actin-binding protein, drebrin, from hippocampal synapses in Alzheimer's disease. J Neurosci Res 1996; 43: 87-92.

9. Shim KS, Lubec G. Drebrin, a dendritic spine protein, is manifold decreased in brains of patients with Alzheimer's disease and Down syndrome. Neurosci Lett 2002; 324: 209212.

10. Ishizuka Y, Hanamura K. Drebrin in Alzheimer's disease. Adv Exp Med Biol 2017; 1006: 203-223.

11. Sekino Y, Tanaka S, Hanamura K, et al. Activation of N-methyl-D-aspartate receptor induces a shift of drebrin distribution: disappearance from dendritic spines and appearance in dendritic shafts. Mol Cell Neurosci 2006; 31: 493-504.

12. Mizui T, Sekino Y, Yamazaki H, et al. Myosin II ATPase activity mediates the long-term potentiation-induced exodus of stable F-actin bound by drebrin A from dendritic spines PLoS One 2014; 9: e85367.

13. Franklin KBJ, Paxinos G. The mouse brain in stereotaxic coordinates. Academic Press, 1997.

14. El-Husseini AE, Schnell E, Chetkovich DM, et al. PSD-95 involvement in maturation of excitatory synapses. Science 2000; 290: 1364-1368.

15. Shirao T, Hanamura K, Koganezawa N, et al. The role of drebrin in neurons. J Neurochem 2017; 141: 819-834.

16. Pleasure SJ, Page C, Lee VM. Pure, postmitotic, polarized human neurons derived from NTera 2 cells provide a system for expressing exogenous proteins in terminally differentiated neurons. J Neurosci 1992; 12:1802-1815.

17. Sanchez MC, Benitez A, Ortloff L, et al. Alterations in glutamate uptake in NT2-derived neurons and astrocytes after exposure to gamma radiation. Radiat Res 2009; 171: 41-52.

18. Sanchez MC, Nelson GA, Green LM. Effects of protons and HZE particles on glutamate transport in astrocytes, neurons and mixed cultures. Radiat Res 2010; 174: 669-678.

19. Greene-Schloesser D, Robbins ME. Radiation-induced cognitive impairment--from bench to bedside. Neuro Oncol 2012; 14: 37-44.

20. Kyrkanides S, Moore AH, Olschowka JA, et al. Cyclooxygenase-2 modulates brain inflammation-related gene expression in central nervous system radiation injury. Mol Brain Res 2002; 104:159-169.

21. Moore AH, Olschowka JA, Williams JP, et al. Regulation of prostaglandin E2 synthesis after brain irradiation. Int $\mathrm{J}$ Radiat Oncol Biol Phys 2005; 62: 267-272.

22. Shi L, Adams MM, Long A, et al. Spatial learning and memory deficits after whole-brain irradiation are associated with changes in NMDA receptor subunits in the hippocampus. Radiat Res 2006; 166: 892-899. 